\title{
Optimum Frequency-Domain Partial Response Encoding in OFDM System
}

\author{
Hua Zhang and Ye (Geoffrey) Li, Senior Member, IEEE
}

\begin{abstract}
Time variance of wireless channels destroys the orthogonality among subchannels in orthogonal frequency-division multiplexing (OFDM) systems and causes interchannel interference (ICI), which results in an error floor. In this letter, we study frequency-domain partial-response coding (PRC) for reducing the effect of the ICI. Based on the general expression of the ICI power for OFDM with PRC, the optimum weights for PRC that minimize the ICI power are derived. From the numerical and simulation results, optimum PRC for OFDM can reduce the ICI effectively by 4.0 dB for two-tap PRC and 6.2 dB for three-tap PRC, respectively.
\end{abstract}

Index Terms-Interchannel interference (ICI), orthogonal frequency-division multiplexing (OFDM), partial-response coding (PRC).

\section{INTRODUCTION}

A $\mathrm{S}$ A promising technique for high data-rate transmission [1], orthogonal frequency-division multiplexing (OFDM) has been successfully used in many environments, such as digital audio broadcasting (DAB), digital video broadcasting (DVB), and HyperLAN-II. In a classical OFDM system, the entire channel is divided into many orthogonal subchannels, and information symbols are transmitted in parallel over these subchannels with a long symbol duration to deal with the frequency-selective fading of wireless environments. However, it has been shown [2]-[5] that time variation of wireless channels over an OFDM symbol period destroys orthogonality among subchannels and causes the interchannel interference (ICI). If not compensated for, the ICI will result in an error floor, which increases with Doppler frequency and symbol duration. Several methods [6]-[16] have been proposed to reduce the effect of the ICI. One of the most commonly used methods is frequency-domain equalization [6], [7]. In [6], a pilot symbol assisted frequency-domain equalizer was proposed. In [7], an equalization technique suitable to time-varying multipath channel has been developed. The antenna diversity is an effective way to combat the effects of time variation of wireless channels, hence, can reduce the ICI as shown in [2]. Another way to deal with the ICI is the time-domain windowing in [8] and [9]. In [10], ICI suppression in multiple-input multiple-output (MIMO) OFDM is studied. Recently, a self-ICI-cancellation approach [11] has been proposed, which transmits each symbol over a pair of adjacent subchannels with a $180^{\circ}$ phase shift. This method can suppress the ICI significantly with a reduction

Paper approved by N. Al-Dhahir, the Editor for Space-Time, OFDM, and Equalization of the IEEE Communications Society. Manuscript received April 24, 2002; revised October 23, 2002 and January 13, 2003. This work was supported in part by the National Science Foundation under Grant CCR-0121565, in part by Nortel Network, and in part by a research gift from Hughes Network System.

The authors are with the School of Electrical and Computer Engineering, Georgia Institute of Technology, Atlanta, GA 30332-0250 USA (e-mail: huazhang@ece.gatech.edu; liye@ece.gatech.edu).

Digital Object Identifier 10.1109/TCOMM.2003.814203

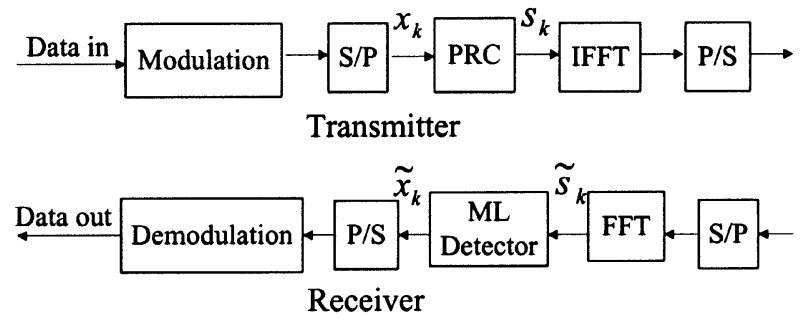

Fig. 1. OFDM system with PRC.

in bandwidth efficiency. Partial-response coding (PRC) in the time domain has been studied for single-carrier systems to reduce the sensitivity to time offset [12] without sacrificing the bandwidth. In the frequency domain, the PRC with correlation polynomial $F(D)=1-D$ was used to mitigate the ICI caused by carrier frequency offset in [13]. In this letter, we study general frequency-domain PRC for suppressing the ICI caused by Doppler frequency shift or carrier frequency offset.

This letter is organized as follows. In Section II, we describe an OFDM system with PRC and derive the exact ICI expression. With the expression, we obtain the optimum weights of PRC based on minimizing the ICI and analyze its performance in Section III. Then, in Section IV, the numerical and simulation results are presented to demonstrate the performance improvement of OFDM systems with optimum PRC.

\section{ICI FOR OFDM WITH PRC}

The baseband model of OFDM with PRC is shown in Fig. 1. At the transmitter, the modulated signal is encoded by PRC. Let $x_{k}$ be the symbols to be transmitted and $c_{i}$ be the weights for PRC with unit norm, i.e.,

$$
\sum_{i=0}^{K-1} c_{i}^{2}=1
$$

where $K$ is the number of weights of PRC. Without loss of generality, we assume $E\left|x_{k}\right|^{2}=1$ and $E\left(x_{k} x_{l}^{*}\right)=0$ for $k \neq l$. Then the transmitted signal at the $k$ th subchannel can be expressed as

$$
s_{k}=\sum_{i=0}^{K-1} c_{i} x_{k-i}
$$

The coded signals can be recovered by a maximum-likelihood (ML) sequence detector [14] at the receiver. The OFDM signal in time domain is

$$
y(t)=\sum_{k} s_{k} e^{j 2 \pi f_{k} t}, \quad 0 \leq t<T_{s}
$$

where $f_{k}=f_{0}+k \Delta f$ is the frequency of the $k$ th subchannel, $\triangle f=1 / T_{s}$ is the subchannel spacing, and $T_{s}$ is the symbol 
duration. After passing through a time-varying channel with the impulse response $h(t, \tau)$, the received signal is

$$
\tilde{y}(t)=\int h(t, \tau) y(t-\tau) d \tau \text {. }
$$

The channel impulse response for the frequency-selective fading channel can be described by

$$
h(t, \tau)=\sum_{l} \gamma_{l}(t) \delta\left(\tau-\tau_{l}\right)
$$

where $\tau_{l}$ is the delay of the $l$ th path, and $\gamma_{l}(t)$ is the corresponding path gain. Here, we assume that the complex stochastic processes $\gamma_{l}(t)$ are independent for different $l$ 's and have the same statistics but different variance $\varepsilon_{l}$. For simplicity, we first consider the flat-fading channel and omit the subscription $l$. Then the received signal becomes

$$
\tilde{y}(t)=\gamma(t) y(t) \text {. }
$$

The demodulated signal can be written as

$$
\tilde{s}_{m}=\frac{1}{T_{s}} \int_{0}^{T_{s}} \tilde{y}(t) e^{-\jmath 2 \pi f_{m} t} d t
$$

Here, the integration is used instead of the discrete Fourier transform (DFT). As indicated in [5], the difference is negligible. It has been derived in [5] that the demodulated signal can be expressed as

$$
\tilde{s}_{m}=a_{0} s_{m}+\sum_{k \neq m} a_{m-k} s_{k}
$$

where $a_{l}$ is defined as

$$
a_{l}=\frac{1}{T_{s}} \int_{0}^{T_{s}} \gamma(t) e^{-\jmath 2 \pi l \triangle f t} d t
$$

Here, $a_{0}$ is the gain of the desired signal and $a_{l}$, for $l \neq 0$, represents the gain of the interfering signals from other subchannels. For time-invariant channels, $\gamma(t)$ is a constant and $a_{l}=0$ for $l \neq 0$; consequently, there is no ICI. In general, for time-varying channels, $a_{l} \neq 0$ for some $l \neq 0$, the ICI exists.

The total ICI power is defined as

$$
P_{\mathrm{ICI}}=E\left|\sum_{l \neq 0} a_{l} s_{m-l}\right|^{2} .
$$

For OFDM without PRC [5], it is

$$
\tilde{P}_{\mathrm{ICI}}=1-2 \int_{0}^{f_{d}} P(f) \operatorname{sinc}^{2}\left(f T_{s}\right) d f
$$

where $f_{d}$ is the maximum Doppler frequency shift, $P(f)$ is the power spectral density of $\gamma(t)$, and $\operatorname{sinc}(x)=\sin (\pi x) /(\pi x)$.

It is derived in the appendix that $P_{\mathrm{ICI}}$ for OFDM with PRC can be expressed as

$$
P_{\mathrm{ICI}}=1-2 \int_{0}^{f_{d}} P(f) \operatorname{sinc}^{2}\left(f T_{s}\right) d f+I_{\mathrm{PRC}}\left(\mathbf{c}_{K}, f_{d} T_{s}\right)
$$

where

$$
\begin{aligned}
& I_{\mathrm{PRC}}\left(\mathbf{c}_{K}, f_{d} T_{s}\right) \\
& =\int_{0}^{f_{d}} \frac{8 \sin \left(\pi f T_{s}\right)^{2} P(f)}{\pi^{2}}\left(\sum_{k=1}^{K-1} \sum_{i=0}^{K-1-k} \frac{c_{i} c_{i+k}}{k^{2}-f^{2} T_{s}^{2}}\right) d f \\
& =\int_{0}^{f_{d}} \frac{8 \sin \left(\pi f T_{s}\right)^{2} P(f)}{\pi^{2}} I\left(\mathbf{c}_{K}, f T_{s}\right) d f
\end{aligned}
$$

with

$$
\mathbf{c}_{K}=\left[c_{0}, c_{1}, \ldots \ldots, c_{K-1}\right]^{T}
$$

and

$$
I\left(\mathbf{c}_{K}, f T_{s}\right)=\sum_{k=1}^{K-1} \sum_{i=0}^{K-1-k} \frac{c_{i} c_{i+k}}{k^{2}-f^{2} T_{s}^{2}}
$$

\section{OPTIMUM PRC FOR OFDM}

In the previous section, we have introduced OFDM with PRC and derived the expression of the ICI power. In this section, we will investigate optimum PRC weights and analyze its performance.

\section{A. Optimum Weights for PRC}

From (10), the ICI power includes two parts: the ICI power for OFDM without PRC; and $I_{\mathrm{PRC}}\left(\mathbf{c}_{K}, f_{d} T_{s}\right)$ contributed by PRC. Therefore, the only way to reduce the ICI power is to minimize $I_{\mathrm{PRC}}\left(\mathbf{c}_{K}, f_{d} T_{s}\right)$ with respect to $\mathbf{c}_{K}$. In the integral of $I_{\mathrm{PRC}}\left(\mathbf{c}_{K}, f_{d} T_{s}\right)$, the first part, $\left(8 \sin \left(\pi f T_{s}\right)^{2} P(f) / \pi^{2}\right)$, is always positive. So we need only make the last part as small as possible. When $f^{2} T_{s}^{2} \ll 1$, the last part can be approximated as

$$
I\left(\mathbf{c}_{K}, f T_{s}\right) \approx g\left(\mathbf{c}_{K}\right)=\sum_{k=1}^{K-1} \sum_{i=0}^{K-1-k} \frac{c_{i} c_{i+k}}{k^{2}}=\mathbf{c}_{K}^{T} \mathbf{R}_{K} \mathbf{c}_{K}
$$

where $\mathbf{R}_{K}$ is defined as

$$
\mathbf{R}_{K}=\left[\begin{array}{ccccc}
0 & \frac{1}{2} & \frac{1}{8} & \cdots & \frac{1}{2(K-1)^{2}} \\
\frac{1}{2} & 0 & \frac{1}{2} & \cdots & \frac{1}{2(K-2)^{2}} \\
\frac{1}{8} & \frac{1}{2} & 0 & \cdots & \frac{1}{2(K-3)^{2}} \\
\vdots & \vdots & \vdots & \ddots & \vdots \\
\frac{1}{2(K-1)^{2}} & \frac{1}{2(K-2)^{2}} & \frac{1}{2(K-3)^{2}} & \cdots & 0
\end{array}\right] .
$$

From (12), it is clear that the optimum $\mathbf{c}_{K}$ that minimizes the ICI is the normalized eigenvector of $\mathbf{R}_{K}$ corresponding to the smallest eigenvalue. In this case

$$
I_{\min }\left(\mathbf{c}_{K}, f T_{s}\right) \approx g_{\min }\left(\mathbf{c}_{K}\right)=\lambda_{0}^{(K)}
$$

where $\lambda_{0}^{(K)} \leq \lambda_{1}^{(K)} \leq \cdots \leq \lambda_{K-1}^{(K)}$ are the ordered eigenvalues of $\mathbf{R}_{K}$.

When $K \rightarrow \infty$, we can obtain the limit of $g_{\min }\left(\mathbf{c}_{K}\right)$ using [17, Corollary 4.2]

$$
\lim _{K \rightarrow \infty} g_{\min }\left(\mathbf{c}_{K}\right)=\min _{\lambda \in \Re} f(\lambda)
$$

where $f(\lambda)$ is defined as

$$
f(\lambda)=\sum_{k \neq 0} \frac{1}{2 k^{2}} e^{\jmath k \lambda}
$$

The minimum of $f(\lambda)$ can be found by setting its derivative with respect to $\lambda$ to be zero, that is

$$
\frac{d}{d \lambda} f(\lambda)=-\sum_{k=1}^{\infty} \frac{1}{k} \sin (k \lambda)=0 .
$$

Solving (15) for $\lambda$ and substituting it into (13) leads to

$$
\lim _{K \rightarrow \infty} g_{\min }\left(\mathbf{c}_{K}\right)=\sum_{k=1}^{\infty} \frac{(-1)^{k}}{k^{2}}=-\frac{\pi^{2}}{12} \text {. }
$$


Once $g_{\min }\left(\mathbf{c}_{K}\right)$ is found, the total ICI power for OFDM with optimum PRC can be calculated using (10).

\section{B. Performance Analysis}

The exact expression of the ICI is too complicated to provide much insight. In many cases, it is difficult to calculate the ICI because the exact power spectrum is not available. Here, we derive an approximate expression using a similar method to [5].

If $f T_{s} \ll 1$, we have the following approximation:

$$
\sin ^{2}\left(\pi f T_{s}\right) \approx\left(\pi f T_{s}\right)^{2} .
$$

Substituting (12) and (17) into (11), we can obtain the following expression:

$$
\begin{aligned}
I_{\mathrm{PRC}}\left(\mathbf{c}_{K}, f_{d} T_{s}\right) & \approx \int_{0}^{f_{d}} \frac{8\left(\pi f T_{s}\right)^{2}}{\pi^{2}} P(f) g\left(\mathbf{c}_{K}\right) d f \\
& =4 \alpha g\left(\mathbf{c}_{K}\right)\left(f_{d} T_{s}\right)^{2}
\end{aligned}
$$

where $\alpha$ is defined as

$$
\alpha=\frac{2}{T_{s}^{2}} \int_{0}^{f_{d}} f^{2} P(f) d f
$$

which is dependent on the spectral density of $\gamma(t)$. It is calculated in [5] that $\alpha=1 / 2$ for the classical Doppler spectrum (Jakes' model) and $\alpha=1$ for OFDM with carrier offset.

Using [5, eq. (3.10)] and (18), we can get an approximate expression of the ICI for PRC signals as

$$
P_{\mathrm{ICI}} \approx\left[\frac{\pi^{2}}{3}+4 g\left(\mathbf{c}_{K}\right)\right] \alpha\left(f_{d} T_{s}\right)^{2}
$$

The expression is much simpler than the exact one. From (18), the total ICI is decided by three factors: $\alpha ; f_{d} T_{s}$; and $g\left(\mathbf{c}_{K}\right)$. $f_{d} T_{s}$ can be regarded as the normalized Doppler frequency shift. $g\left(\mathbf{c}_{K}\right)$ represents the ICI reduction due to PRC. For OFDM without PRC [5]

$$
\tilde{P}_{\mathrm{ICI}} \approx \frac{\pi^{2}}{3} \alpha\left(f_{d} T_{s}\right)^{2} .
$$

Then, the performance gain of PRC is

$$
\begin{aligned}
\operatorname{Gain}(\mathrm{dB}) & =\tilde{P}_{\mathrm{ICI}}(\mathrm{dB})-P_{\mathrm{ICI}}(\mathrm{dB}) \\
& \approx-10 \log _{10}\left[1+\frac{12}{\pi^{2}} g\left(\mathbf{c}_{K}\right)\right] .
\end{aligned}
$$

Table I lists the optimum weights, the corresponding $g\left(\mathbf{c}_{K}\right)$, and the performance gain, according to the above results. From the table, the value of $g\left(\mathbf{c}_{K}\right)$ is close to the limit when $K=4$.

In the above discussion, we obtained the optimum PRC and analyzed its performance for flat-fading channels. For frequency-selective fading channels, it is usually assumed that a cyclic extension is inserted ahead of each OFDM symbol to combat the interblock interference (IBI). Then, the expression of the ICI for frequency-selective fading channels can be derived in a similar way

$$
P_{\mathrm{ICI}}=1-2 \int_{0}^{f_{d}} P(f) \operatorname{sinc}^{2}\left(f T_{s}\right) d f+\tilde{I}_{\mathrm{PRC}}\left(\mathbf{c}_{K}, f_{d} T_{s}\right)
$$

where

$$
\tilde{I}_{\mathrm{PRC}}\left(\mathbf{c}_{K}, f_{d} T_{s}\right)=\int_{0}^{f_{d}} \frac{8 \sin \left(\pi f T_{s}\right)^{2} P(f)}{\pi^{2}} \tilde{I}\left(\mathbf{c}_{K}, f T_{s}\right) d f
$$

TABLE I

\begin{tabular}{c|c|c|c}
\hline$K$ & $\mathbf{c}_{K}$ & $-g\left(\mathrm{c}_{K}\right)$ & $\mathrm{Gain}(\mathrm{dB})$ \\
\hline 1 & 1 & 0 & 0 \\
\hline 2 & $0.7071,-0.7071$ & 0.5000 & 4.066 \\
\hline 3 & $-0.4775,0.7376,-0.4775$ & 0.6474 & 6.719 \\
\hline 4 & $-0.3501,0.6144,-0.6144,0.3501$ & 0.7137 & 8.786 \\
\hline$\infty$ & & 0.8225 & - \\
\hline
\end{tabular}

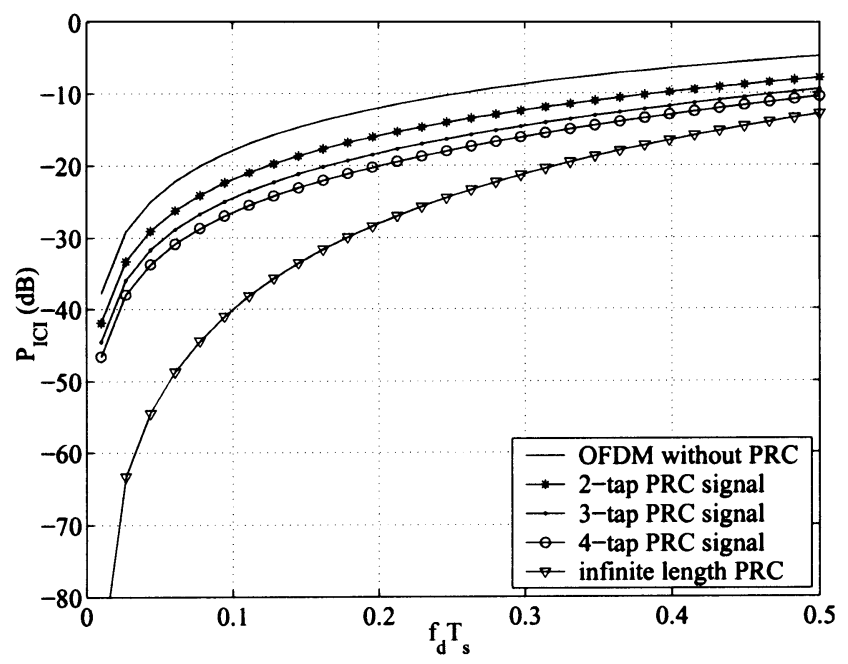

Fig. 2. Comparison of the ICI power due to Doppler frequency shift.

and

$$
\tilde{I}\left(\mathbf{c}_{K}, f T_{s}\right)=\sum_{k=1}^{K-1} \sum_{i=0}^{K-1-k} \sum_{l} \varepsilon_{l} e^{\jmath 2 \pi k \Delta f \tau_{l}} \frac{c_{i} c_{i+k}}{k^{2}-f^{2} T_{s}^{2}} .
$$

Usually, the path delay $\tau_{l}$ is much smaller than an OFDM symbol duration. Then for small-size PRC, the term $\varepsilon_{l} e^{\jmath 2 \pi k \Delta f \tau_{l}}$ approximates to constant. Therefore, the optimum PRC with small size obtained for flat-fading channels are applicable to the frequency-selective fading channel.

\section{NuMERICAL AND Simulation RESUlTS}

In this section, we present some numerical and simulation results to show the performance improvement of OFDM with optimum PRC.

\section{A. Numerical Results}

To examine the performance improvement, we compare the ICI value of OFDM with and without PRC.

Fig. 2 presents a comparison of ICI power for the classical Doppler spectrum (Jakes' model)

$$
P_{J}(f)= \begin{cases}\frac{1}{\pi f_{d}} \frac{1}{\sqrt{1-\left(\frac{f}{f_{d}}\right)^{2}}}, & \text { if }|f|<f_{d} \\ 0, & \text { else. }\end{cases}
$$

From the figure, the ICI power is reduced by about $4.0 \mathrm{~dB}$ for two-tap and about $6.2 \mathrm{~dB}$ for three-tap PRC, respectively.

The optimum PRC can also reduce the ICI due to carrier offset as shown in Fig. 3. There is about 4.5-dB improvement for two-tap PRC. 


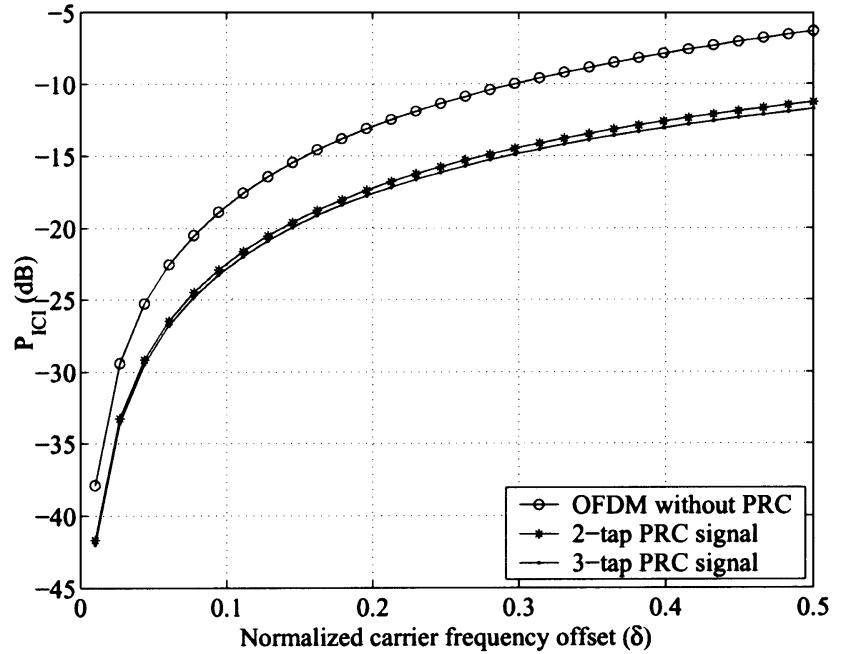

Fig. 3. Comparison of the ICI power due to carrier offset.

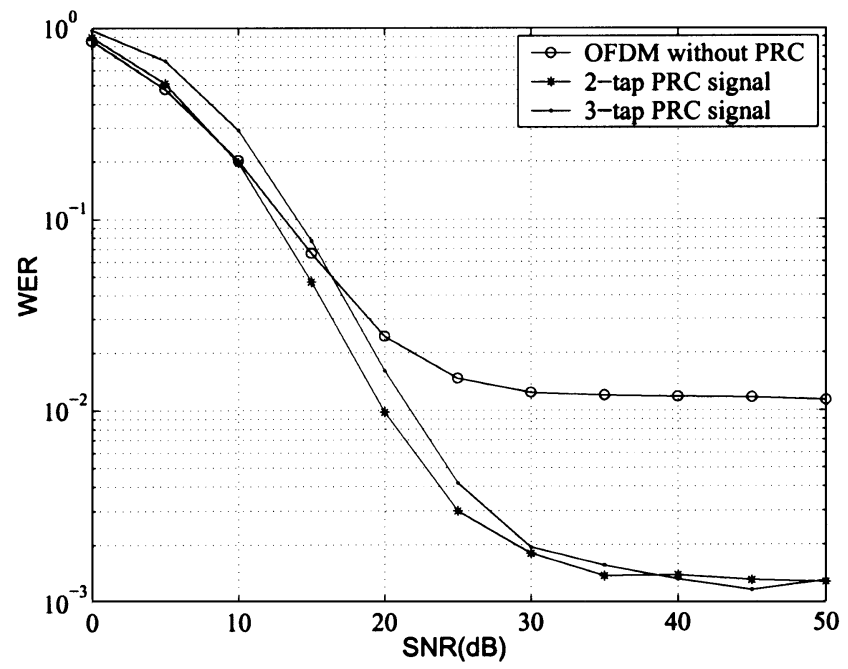

Fig. 4. WER of OFDM with and without PRC $\left(f_{d} T_{s}=0.1\right)$.

\section{B. Simulation Results}

Here, we present our simulation results for the performance of PRC. The system model and parameters used in our simulation are the same as those in [20]. The entire channel bandwidth $(800 \mathrm{kHz})$ is divided into 128 subchannels. 120 tones at the middle are used to transmit data. The rest tones on each side are used as guard tones. Quaternary phase-shift keying (QPSK) with coherent demodulation is used. A $(40,20)$ Reed-Solomon (R-S) code, with each code symbol consisting of three QPSK symbols grouped in frequency, is used so that each block forms an $\mathrm{R}-\mathrm{S}$ codeword. The noise is assumed to be white Gaussian with zero-mean and variance $\sigma_{n}^{2}$, then the signal-to-noise ratio (SNR) is $1 / \sigma_{n}^{2}$. The time-varying fading channel is generated using Jakes' model. A cyclic prefix of length larger than the delay spread is used to avoid IBI.

Fig. 4 shows word-error rate (WER) versus SNR for a hillyterrain (HT) channel. From the figure, OFDM with PRC has some performance loss at lower SNR. However, for the system at a high SNR, the ICI is the dominant impairment and PRC has better performance than without PRC. And the error floor

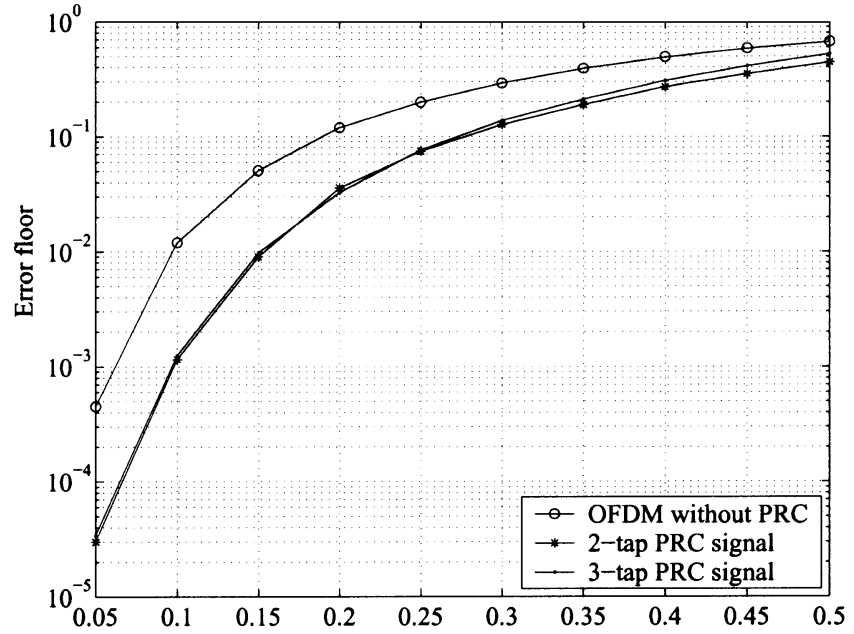

Fig. 5. Error-floor comparison for OFDM signal with and without PRC.

due to Doppler frequency shift is reduced from $10^{-2}$ to $10^{-3}$. Because PRC has some performance loss when $K>2$, as for time-domain PRC [12], the performance of three-tap PRC is not as good as that of two-tap PRC, though three-tap PRC has better performance for ICI suppression.

The error floors versus normalized Doppler frequency shift are shown in Fig. 5. From the figure, to ensure WER below $1 \%$, the maximum tolerable Doppler frequency shift for OFDM without PRC is about $10 \%$ of subchannel spacing, and it is relaxed to be $15 \%$ for OFDM with two-tap or three-tap PRC.

\section{CONCLUSION}

In this letter, we investigated frequency-domain PRC to reduce the ICI caused by time variation of wireless channels. The optimum weights for PRC that minimizes the ICI power are obtained. The numerical and simulation results show that PRC effectively reduces the error floor caused by Doppler frequency shift or carrier offset. Even though our discussions are for flatfading channels, the obtained optimum PRCs are also applicable to the frequency-selective channels.

\section{APPENDIX \\ DERIVATION OF ICI}

Here, we derive the ICI power due to Doppler frequency shift for OFDM with PRC. First, we calculate the autocorrelation of $a_{l}$, that is

$$
\begin{aligned}
& E\left(a_{m} a_{n}^{*}\right)= \\
& E\left\{\frac{1}{T_{s}} \int_{0}^{T_{s}} \gamma(t) e^{-j 2 \pi m \Delta f t} d t \frac{1}{T_{s}} \int_{0}^{T_{s}} \gamma^{*}(\tau) e^{-j 2 \pi n \Delta f \tau} d \tau\right\}
\end{aligned}
$$

where $m \neq n$. By (7) and [18, eq. (10-50)]

$$
E\left(a_{m} a_{n}^{*}\right)=\int_{-1}^{1} r\left(T_{s} x\right) \frac{-\sin [\pi(m-n)|x|]}{\pi(m-n)} e^{-j 2 \pi(m+n / 2) x} d x
$$


where $r(\tau)$ is the autocorrelation of $\gamma(t)$. Then the ICI power is then

$$
\begin{aligned}
P_{\mathrm{ICI}}= & E\left|\sum_{l \neq 0} a_{l} s_{m-l}\right|^{2} \\
= & E\left|\sum_{l \neq 0} a_{l} \sum_{i=0}^{K-1} c_{i} x_{m-l-i}\right|^{2} \\
= & \sum_{n \neq i} \sum_{i=0}^{K-1} c_{i}^{2} E\left|a_{n-i}\right|^{2} \\
& +2 \sum_{\substack{n \neq i \\
n \neq i+k}} \sum_{k=0}^{K-1} \sum_{i=0}^{K-1-k} c_{i} c_{i+k} E\left(a_{n-i} a_{n-i-k}^{*}\right) \\
= & \sum_{l \neq 0} E\left|a_{l}\right|^{2}+2 \sum_{k=1}^{K-1} \sum_{i=0}^{K-1-k} c_{i} c_{i+k} T_{k}
\end{aligned}
$$

$$
P_{\mathrm{ICI}}=1-2 \int_{0}^{f_{d}} P(f) \operatorname{sinc}^{2}\left(f T_{s}\right) d f+I_{\mathrm{PRC}}\left(c_{K}, f_{d} T_{s}\right)
$$

where

$$
\begin{aligned}
& I_{\mathrm{PRC}}\left(\mathbf{c}_{K}, f_{d} T_{s}\right)= \\
& \int_{0}^{f_{d}} \frac{8 \sin \left(\pi f T_{s}\right)^{2} P(f)}{\pi^{2}}\left(\sum_{k=1}^{K-1} \sum_{i=0}^{K-1-k} \frac{c_{i} c_{i+k}}{k^{2}-f^{2} T_{s}^{2}}\right) d f .
\end{aligned}
$$

\section{REFERENCES}

[1] L. J. Cimini, Jr., "Analysis and simulation of a digital mobile channel using orthogonal frequency-division multiplexing," IEEE Trans. Commun., vol. COM-33, pp. 665-765, July 1985.

[2] M. Russell and G. L. Stüber, "Interchannel interference analysis of OFDM in a mobile environment," in Proc. VTC'95, 1995, pp. 820-824.

where we have used the identity $\sum_{i=0}^{K-1} c_{i}^{2}=1$ and the definition

$$
T_{k}=\sum_{n} E\left(a_{n-i} a_{n-i-k}^{*}\right)-E\left(a_{0} a_{-k}^{*}\right)-E\left(a_{k} a_{0}^{*}\right)
$$

Substituting (A.2) into (A.3), we have

$$
\begin{aligned}
T_{k}=\int_{-1}^{1} r\left(T_{s} x\right) \frac{-\sin (\pi k|x|)}{\pi x} \\
\times\left[\sum_{n} e^{-\jmath 2 \pi(2(n-i)-k / 2) x}-2 \cos (\pi k x)\right] d x .
\end{aligned}
$$

From [19, eqs. (3.2) and (3.5)]

$$
\sum_{n=-\infty}^{\infty} e^{-\jmath 2 \pi n x}=\sum_{m=-\infty}^{\infty} \delta(x-m) .
$$

Consequently

$$
\begin{aligned}
T_{k} & =\int_{-1}^{1} r\left(T_{s} x\right) \frac{-\sin (\pi k|x|)}{\pi k}\left[\sum_{m} \delta(x-m) e^{\jmath \pi k x}-2 \cos (\pi k x)\right] d x \\
& =\int_{-1}^{1} r\left(T_{s} x\right) \frac{2 \sin (\pi k|x|) \cos (\pi k x)}{\pi k} d x \\
& =\int_{-1}^{1} \frac{\sin (2 \pi k|x|)}{\pi k}\left[2 \int_{0}^{f_{d}} P(f) \cos \left(2 \pi f T_{S} x\right) d f\right] d x \\
& =\int_{0}^{f_{d}} \frac{4 \sin ^{2}\left(\pi f T_{s}\right) P(f)}{\pi^{2}\left(k^{2}-f^{2} T_{s}^{2}\right)} d f .
\end{aligned}
$$

From [5]

$$
\sum_{l \neq 0} E\left|a_{l}\right|^{2}=1-2 \int_{0}^{f_{d}} P(f) \operatorname{sinc}^{2}\left(f T_{s}\right) d f
$$

3] P. Robertson and S. Kaiser, "The effects of Doppler spreads in OFDM(A) mobile radio systems," in Proc. VTC'99-Fall, 1999, pp. 329-333.

[4] - "Analysis of the loss of orthogonality through Doppler spread in OFDM systems," in Proc. GLOBECOM'99, vol. 1b, 1999, pp. 701-706.

[5] Y. (G.) Li and L. J. Cimini, Jr., "Bounds on the interchannel interference of OFDM in time-varying impairments communications," IEEE Trans. Commun., vol. 49, pp. 401-404, Mar. 2001.

[6] J. Ahn and H. S. Lee, "Frequency-domain equalization of OFDM signal over frequency nonselective Rayleigh fading channels," Electron. Lett., vol. 29, pp. 1476-1477, Aug. 1993.

[7] W. G. Jeon, K. H. Chang, and Y. S. Cho, "An equalization technique for orthogonal frequency-division multiplexing systems in time-variant multipath channels," IEEE Trans. Commun., vol. 47, pp. 27-32, Jan. 1999.

[8] C. Muschallik, "Improving an OFDM reception using an adaptive Nyquist windowing," IEEE Trans. Consumer Electron., vol. 42, pp. 259-269, Aug. 1996.

[9] J. Armstrong, "Analysis of new and existing methods of reducing intercarrier interference due to carrier frequency offset in OFDM," IEEE Trans. Commun., vol. 47, pp. 365-369, Mar. 1999.

[10] S. Diggavi, N. Al-Dhahir, and A. Stamoulis, "Intercarrier interference in MIMO OFDM," in Proc. IEEE ICC, Oct. 2002, pp. 485-489.

[11] Y. Zhao and S. G. Häggman, "Intercarrier interference self-cancellation scheme for OFDM mobile communication systems," IEEE Trans. Commun., vol. 49, pp. 1185-1191, July 2001.

[12] J. G. Proakis, Digital Communications, 3rd ed, Y. Zhao, J. D. Leclercq, and S. G., Eds. New York: McGraw-Hill, 1995.

[13] Y. Zhao and S. G. Häggman, "Intercarrier interference compression in OFDM communication systems by using correlative coding," IEEE Commun. Lett., vol. 2, pp. 214-216, Aug. 1998.

[14] G. D. Forney, Jr., "Maximum-likelihood sequence estimation of digital sequences in the presence of intersymbol interference," IEEE Trans. Inform. Theory, vol. IT-18, pp. 363-378, May 1972.

[15] P. H. Moose, "A technique for orthogonal frequency-division multiplexing frequency offset correction," IEEE Trans. Commun., vol. 42, pp. 2908-2914, Oct. 1994.

[16] S. Alam, "A general coding method to minimize intercarrier interference in OFDM mobile communication systems," in Proc. Int. Wireless and Telecommunications Symp./Exhibition (IWTS'97), vol. 1, Malaysia, May 1997, pp. 231-235.

[17] M. G. Robert. Toeplitz and Circulant Matrices: A Review. Stanford Univ., Stanford, CA. [Online]. Available: http://ee.stanford.edu/gray/toeplitz.pdf

[18] A. Papoulis, Probability, Random Variables, and Stochastic Processes, 3rd ed. New York: McGraw-Hill, 1991.

[19] A. V. Oppenheim and R. W. Schafer, Discrete-Time Signal Processing. Englewood Cliffs, NJ: Prentice-Hall, 1989.

[20] Y. (G.) Li, L. J. Cimini, Jr., and N. R. Sollenberger, "Robust channel estimation for OFDM systems with rapid dispersive fading channels," IEEE Trans. Commun., vol. 46, pp. 902-915, July 1998. 